\title{
Estado de la cuestión de los recursos didácticos en los programas del español como lengua extranjera (ELE) en Bogotá y Medellín
}

\author{
Diana Marcela Bernal León ${ }^{1}$ \\ Yuribia Andrea Caro ${ }^{2}$ \\ Orlando Rodríguez Ochoa ${ }^{3}$ \\ Gustavo Jaramillo Cardona ${ }^{4}$ \\ José Orlando Gómez Salazar 5
}

Recibido: 10-04-2019

Aceptado: 04-07-2019

1. Licenciada en Lengua Castellana, Inglés y Francés de la Universidad de la Salle; especialista en Pedagogía y Docencia Universitaria; magíster en Educación de la Universidad Externado de Colombia. Docente de tiempo completo del Instituto de Lenguas Fray Bernardo de Lugo O.P., de la Universidad Santo Tomás de Bogotá, adscrita a la línea de investigación "Lenguaje, Pensamiento y Educación" del grupo Ustalearning, reconocido en Colciencias.

Correo electrónico: dianabernall@usantotomas.edu.co

ORCID: https://orcid.org/0000-0001-5733-6531

Google Scholar: https://scholar.google.es/citations?user=TXnyUmAAAAAJ\&hl=es

2. Licenciada en Español y Filología Clásica de la Universidad Nacional de Colombia; magíster en Literatura Latinoamericana de la Pontificia Universidad Javeriana. Docente de tiempo completo del Instituto de Lenguas Fray Bernardo de Lugo O.P., de la Universidad Santo Tomás de Bogotá, adscrita a la línea de investigación "Lenguaje, Pensamiento y Educación" del grupo Ustalearning, reconocido en Colciencias.

Correo electrónico: yuribiacaro@usantotomas.edu.co

ORCID: https://orcid.org/0000-0001-9151-9681

Google Scholar: https://scholar.google.es/citations?hl=es\&user=JETenR8AAAAJ

3. Licenciado en Español e Inglés de la Universidad Pedagógica Nacional; magíster en Lingüística Aplicada para la Enseñanza de Inglés como Lengua Extranjera de la Universidad Distrital Francisco José de Caldas. Docente de tiempo completo del Instituto de Lenguas Fray Bernardo de Lugo O.P., de la Universidad Santo Tomás de Bogotá, adscrita a la línea de investigación "Lenguaje, Pensamiento y Educación" del grupo Ustalearning reconocido en Colciencias.

Correo electrónico: edgarochoa@usantotomas.edu.co

ORCID: https://orcid.org/0000-0002-0785-4530

Google Scholar: https://scholar.google.es/citations?hl=es\&user=GsEl7tQAAAAJ\&view_op=list_ works\&sortby $=$ title

4. Licenciado en Lenguas Modernas (Inglés-Francés) de la Universidad Pontificia Bolivariana; magíster en Lenguas Modernas (Didactología de Lenguas y Cultura) de la Universidad de París III. Pertenece al grupo de investigación "Lengua y Cultura" de la Universidad Pontificia Bolivariana. CvLAC: http://scienti.colciencias.gov.co:8081/cvlac/visualizador/generarCurriculoCv.do?cod $\mathrm{rh}=0001467487$

Correo electrónico: gustavo.jaramillo@upb.edu.co ORCID: https://orcid.org/0000-0002-1746-1669

5. Licenciado en Lenguas Modernas de la Universidad del Valle; magíster en "Teaching English as a Second Language" de Northern Arizona University; doctor en Lenguas Modernas de Wayne State University. Pertenece al grupo de investigación "Lengua y Cultura" de la Universidad Pontificia Bolivariana.

CvLAC: http://scienti.colciencias.gov.co:8081/cvlac/visualizador/generarCurriculoCv.do?cod_

$\mathrm{rh}=0001467663$

Correo electrónico: jose.gomez@upb.edu.co

ORCID: https://orcid.org/0000-0001-5356-6049 


\title{
Resumen
}

Este artículo presenta un estado de la cuestión de los recursos didácticos en español como lengua extranjera (ELE) en los programas de Bogotá y Medellín, como resultado de un trabajo investigativo de cooperación académica interinstitucional entre la Universidad Santo Tomás y la Universidad Pontificia Bolivariana. La primera sección ofrece una aproximación al contexto actual de ELE en Colombia y una presentación de los recursos didácticos utilizados en los programas de ELE en las ciudades mencionadas. La segunda explica el trabajo investigativo realizado, que inicia con el proceso de selección y análisis documental de textos de ELE. Posteriormente, se discuten los hallazgos en torno a las tendencias en el uso de los recursos de didácticos de ELE; luego, se compilan y se explican brevemente varias publicaciones resultado de diferentes trabajos investigativos enfocados al diseño de materiales didácticos de ELE en el país para, finalmente, presentar las conclusiones del análisis realizado.

Palabras clave: Colombia, competencia intercultural, español como lengua extranjera (ELE), recursos didácticos.

\section{Status of teaching resources in the Spanish as a foreign language (SFL) programs in Bogotá and Medellín}

\begin{abstract}
This article reflects on the upheaval and changes in the school iThis article reports on the status of teaching resources in Spanish as a foreign language (SFL) programs in Bogotá and Medellín as a result of an inter-institutional academic cooperation study by the Universidad Santo Tomás and the Universidad Pontificia Bolivariana.
\end{abstract}


The first section looks at the current context of SFL in Colombia and the teaching resources used in SFL programs in the mentioned cities. The second explains the research work carried out, which begins with the process of selection and documentary analysis of SFL texts and then discusses the trends in the use of SFL teaching resources. Finally, the article compiles and briefly explains several research papers focused on the design of SFL teaching materials in the country and present conclusions.

Keywords: Colombia, intercultural competence, Spanish as a foreign language (SFL), teaching resources.

\section{Introducción}

Este artículo es el resultado de un trabajo investigativo realizado por los profesores investigadores de español como lengua extranjera (ELE), de la Universidad Santo Tomás y la Universidad Pontificia Bolivariana, como una contribución a la comunidad académica sobre las implicaciones del uso de recursos didácticos en las aulas de ELE. El estudio presenta información frente a la ausencia de recursos con aspectos culturales colombianos para las prácticas docentes en el país.

Con el objetivo de consolidar los datos encontrados en el desarrollo de la investigación, junto con la información existente adelantada por otros grupos de investigación de ELE en el país en los últimos años que permitan lograr un corpus coherente, el grupo investigador organizó este artículo a la luz de los objetivos del proyecto: 1) caracterizar los recursos didácticos en la enseñanza de ELE en los programas de la Universidad Santo Tomás y la Universidad Pontificia Bolivariana de Medellín; 2) establecer los recursos didácticos que se emplean en el aula ELE en las ciudades de Bogotá y Medellín, y 3) determinar cómo las prácticas y recursos empleados por el docente contribuyen en la inclusión del componente cultural colombiano. 
La primera sección presenta una visión actualizada del ámbito de ELE en Colombia, a través del abordaje de algunos de los elementos que lo conforman: políticas nacionales, redes de participación nacional, programas, convocatorias, entre otros.

Por otro lado, con el fin de cumplir con los objetivos planteados, en la segunda sección se resumen los recursos didácticos más utilizados en los programas de ELE de las universidades pertenecientes al portal Spanish in Colombia, resultado de encuestas realizadas a los coordinadores de programas y representantes de editoriales en el país. En esta sección se presenta el proceso de selección de textos, la revisión de tales recursos a través de matrices de análisis y la triangulación de la información obtenida a la luz de las categorías eje del estudio. Finalmente, esta sección presenta una discusión de los hallazgos y tendencias encontradas en torno al uso de recursos didácticos en las aulas del ELE.

Una de las preocupaciones que encontramos al iniciar nuestro proyecto se relaciona con la pertinencia del programa de ELE en las diferentes instituciones. Por esta razón, en el momento de consolidar el soporte teórico de nuestro estudio, consultamos estudios enfocados hacia el diseño de los programas de lenguas extranjeras. En la tercera sección, se consolida una etapa importante del proyecto investigativo, que aborda los tipos de estudios, así como algunas publicaciones e investigaciones que se han hecho con respecto a ELE. Allí, se hizo una recopilación con comentarios sobre los trabajos investigativos que se han adelantado en algunas instituciones educativas del país. Vale la pena mencionar que el material de investigación de ELE es menos común en comparación con el que se produce respecto de la enseñanza de otras lenguas extranjeras como el inglés. Sin embargo, tanto el número de producciones investigativas como los grupos dedicados a esta tarea en ELE se han incrementado significativamente en el país y se prevé un aumento mayor en los próximos años. 


\section{Contextos}

\section{Contexto de ELE en Colombia}

Según los datos del Instituto Cervantes en su informe El español: una lengua viva (2018), en la actualidad más de 577 millones de personas hablan español como lengua nativa, segunda y extranjera; además, es la segunda lengua del mundo por número de hablantes nativos y la segunda lengua de comunicación internacional. De igual manera, la cifra de estudiantes internacionales que aprenden español como lengua extranjera está en aumento: más de 21 millones de alumnos estudian español como lengua extranjera en el 2018 y, en concreto, la cifra se acerca a los 22 millones de estudiantes en el mundo.

Dado este contexto, y con el objeto de promocionar el país como destino para la enseñanza y aprendizaje de la lengua española, así como la difusión de la cultura colombiana, el Gobierno de Colombia creó en 1942 el Instituto Caro y Cuervo, y en el primer periodo del gobierno de Juan Manuel Santos esta institución se haría cargo de las estrategias para llevar a cabo el punto 64 del Plan de Gobierno que, precisamente, consistía en impulsar a Colombia como un destino para el aprendizaje del español como lengua extranjera. Desde entonces, el Instituto se ha destacado como un organizador de eventos para la promoción de la lengua española en el país (Presidencia de la Republica, 2010).

A partir de esta política, se han presentado iniciativas en torno a la creación de programas de apoyo en la formación y capacitación de los docentes de ELE, así como en la participación en redes y eventos internacionales en pro de impulsar el español como servicio educativo. En esa misma línea, el Ministerio Nacional de Cultura ha impulsado como estrategia nacional las convocatorias de Estímulos desde el 2015, dentro de las cuales se encuentra una beca de estímulo a la creación de materiales de ELE con componente cultural colombiano. 
Por otra parte, varias universidades del país han abierto programas e, incluso, departamentos enteros, denominados centros o institutos, dedicados a la enseñanza y al fomento de ELE. Adicionalmente, en los últimos años se han creado redes de cooperación, como la Red Académica para el Desarrollo del Español como Lengua Extranjera (EnRedELE), fundada en el 2007 por iniciativa de nueve instituciones de educación superior que cuentan con programas de español como lengua extranjera dentro de sus ofertas académicas. Igualmente, desde el Instituto Caro y Cuervo se creó el portal Spanish in Colombia, como una estrategia de promoción y mercadeo diseñada para visibilizar la oferta de programas de ELE en el exterior. Esta red está conformada por más de veinte universidades en siete ciudades de Colombia (Barranquilla, Bogotá, Bucaramanga, Cali, Cartagena, Manizales y Medellín).

Otra estrategia gubernamental es el programa ELE-Focalae, que surgió en el 2013, en el marco de las reuniones del Foro de Cooperación América Latina-Asia del Este-Focalae, conformado por 18 países de América Latina y 16 de Asia del Este. En este programa, las autoridades de los países asiáticos solicitaron el apoyo de Colombia para fortalecer sus competencias en español, teniendo en cuenta el crecimiento del flujo de turistas hispanoparlantes y la calidad lingüística del español de Colombia, que tiene reconocimiento mundial. (Agencia Presidencial de Cooperación Internacional Colombia, 2018).

Por último, varias instituciones del país, gracias a la "explosión" del campo de ELE, han trabajado cooperativamente, con el fin de seguir las tendencias globales del mundo hispánico, tal y como lo presentan Jaramillo, Naranjo y González (2011).

\section{Estado de la cuestión}

Una de las preocupaciones más importantes del grupo investigador durante el desarrollo de la primera fase del proyecto se centró en conocer los recursos didácticos utilizados en los programas de 
ELE en Bogotá y Medellín. Para lograr este objetivo, se revisó la literatura sobre uso de materiales en Colombia en los últimos años, a continuación, se resumen los hallazgos más relevantes.

Para el 2010, González, en su estudio investigativo titulado Enseñanza del español como lengua extranjera: Bibliografía comentada, caracteriza los materiales instruccionales más utilizados en los primeros programas de ELE en el país, destacando: Progresos: Curso Intermedio de Español; Método de español para extranjeros: Prisma Continúa Nivel A2, Prisma Progresa Novel B1; iHola Amigos!: Curso de español para extranjeros, Tradición y cambio: Lecturas sobre la cultura Latinoamericana Contemporánea; Curso de español para extranjeros: Gente 1; Y ahora la gramática: Nivel Principiante; Mosaicos: Spanish as a world language", "Civilización y cultura. Intermediate Spanish Series", y Pocas palabras no bastan: Actividades de comprensión y expresión de español para extranjeros.

Hacia el 2011, García, García y Buitrago, en su artículo denominado "Estado del Arte de ELE en Colombia, una mirada holística: metodologías y enfoques, material didáctico, variedades lingüísticas y dificultades en el área", hacen un resumen de los libros de texto que se utilizan en algunas instituciones colombianas: Aula Latina, con $60 \%$ (México); Aula Internacional, con $20 \%$ (España); Nuevo Ven, con $10 \%$ (España); Gente, con $5 \%$ (España), y De Dos en Dos, con $5 \%$ (España). Por otro lado, es hasta el 2016, cuando Díaz, en su artículo titulado "Estado del arte del español como lengua extranjera (ELE) en Bogotá: cursos de ELE que nacen y se hacen" de la Universidad de Los Andes, menciona el libro de texto Enlace, de origen colombiano. Esto permite afirmar que ha habido un evidente predominio de material de origen español y de otros países (México), mas no de origen y contexto colombiano. Además, ninguna de estas series ofrece material didáctico complementario que el docente pueda aplicar en sus clases.

En la revisión de la literatura también se encontraron nuevas apuestas, como la tesis doctoral titulada El aprendizaje en una comunidad en internet de profesores de español como segunda lengua. Estudio etnográfico virtual de la comunidad Todoele, de Reyes-An- 
gona (2016), de la Universidad Complutense de Madrid. En este estudio se citan los Massive Online Open Courses (MOOC) y se da cuenta de cómo las universidades iniciaron este tipo de proyectos hacia el 2012. Entre los sitios web que ofrecen estos cursos de libre acceso, se encuentran edX, Coursera y Udacity. También al finalizar su estudio etnográfico, el autor se enfoca en la creación y uso del portal Todoele, creado en julio del 2007 por iniciativa de Jesús Suárez, quien ya había creado en el 2002 el portal Todoele (www. todoele.net), como un recurso que sirve para "recopilar, organizar y compartir los recursos e informaciones que un profesor de español necesita para desarrollar su trabajo" (Suárez, entrevista online, citado en CContinuum, 2014). Sin embargo, al explorar el sitio web, el acceso al material didáctico con componente cultural colombiano es escaso.

En el contexto local, nuevas propuestas investigativas se orientan a la creación de recursos didácticos con inclusión de las tecnologías. Tal es el caso de la Maestría en Lingüística Aplicada del ELE, de la Pontificia Universidad Javeriana, donde ya se encuentra el trabajo investigativo "Vamos a turistear aprendiendo español: propuesta didáctica para el aprendizaje de léxico y expresiones durante el recorrido turístico en la Catedral de Sal de Zipaquirá" de Rojas (2017), cuyo propósito fue diseñar, producir y aplicar un material didáctico con el cual los guías de la Catedral de Sal de Zipaquirá, encargados de liderar el recorrido turístico a los visitantes extranjeros, enseñen léxico y algunas expresiones útiles para su estadía y recorrido turístico en la Catedral de Sal de Zipaquirá.

Otras propuestas novedosas son las siguientes:

- ELEBogotaap: prototipo de una aplicación móvil para el aprendizaje de expresiones idiomáticas, del 2015, investigación que tuvo como objetivo presentar el desarrollo de un prototipo de aplicación digital que ayuda a los estudiantes de español como segunda lengua a aprender los elementos léxicos, especialmente, los utilizados por las personas que viven en Bogotá. 
- A buen entendedor, estrategias no le faltan: guía didáctica del profesor sobre estrategias metacognitivas para la comprensión auditiva en el aula de ELE, del 2017.

- Propuesta didáctica en ELE para la enseñanza del tema la orientación y el transporte en el contexto bogotano, trabajo que presenta una propuesta didáctica con fundamentos tanto del enfoque por tareas como del enfoque léxico, demostrando sus similitudes y la posibilidad de combinarlos.

Existen varios intentos para sistematizar este material, como es el caso del Ministerio de Cultura en las convocatorias de Estímulos desde el 2015; el Plan Nacional de Gobierno 2010-2014, en su política de "convertir a Colombia en el mayor destino en América Latina para el aprendizaje del español"; las iniciativas que se han presentado en torno a la creación de programas de apoyo en la formación y capacitación de los docentes de ELE; la participación en redes y eventos internacionales con el fin de impulsar el español como servicio educativo, y la elaboración de algunos proyectos de investigación por parte de grupos en instituciones de educación superior, como la Maestría en ELE de Universidad Javeriana, y la Maestría del Español como Segunda Lengua, del Instituto Caro y Cuervo. Sin embargo, los esfuerzos para el diseño de recursos didácticos de ELE en Colombia no han sido suficientes, ni se han sistematizado apropiadamente.

En los últimos años también se encontró registro de nuevos recursos didácticos de apoyo al proceso de ELE, con libros como el de Cardona, Gutiérrez, Delgadillo y Rodríguez (2014), titulado De paseo por Colombia: Material de apoyo para estudiantes de español como lengua extranjera. En el 2015 se publicó la cartilla Bogotá, Vívela en español, que consta de 10 unidades, cada una con un tema, una tarea final y cuatro secciones de temas relacionados con Bogotá y su oferta turística. Esta publicación fue realizada gracias a una alianza cooperativa entre el Instituto Distrital de Turismo (IDT) —que lidera el producto turístico denominado "Learn Spanish in Bogotá" (Aprende español en Bogotá), cuyo objetivo es el de forta- 
lecer el turismo idiomático- y la Pontificia Universidad Javeriana, bajo la coordinación de los investigadores Nancy Agray Vargas y Carlos Rico Troncoso (2015).

Por otra parte, en la revisión de la literatura local, se encontró que entre el 2011 y el 2015 hubo un "auge" en el diseño la apertura de programas de ELE en las universidades e instituciones del país, y de igual manera aparecieron redes para el aprendizaje. Además se encontró que docentes y expertos de ELE en el país, han iniciado el camino en la confección de materiales para la enseñanza de ELE desde el 2004; no obstante, muchos de esos materiales no han sido tenidos en cuenta en los planes curriculares de los programas que se ofrecen en el contexto local. Entre los textos recopilados se encuentran: Español para extranjeros (Calderón-Rivera, 2011); Comuniquémonos en español (Parra-Giraldo \& Rojas-Bernal, 2007), elaborado para hablantes nativos de portugués en Brasil; Gramática General del español como lengua extranjera (CardonaSerrano, 2009); Español para extranjeros, nivel inicial (Velandia, Ussa, \& Waked, 2008), intermedio (Velandia et al., 2010) y avanzado (Velandia et al., 2015); Cartagena Hispana y Multicultural, texto de español para estudiantes extranjeros, niveles básico, intermedio y avanzado (Cabeza, Lobo, \& Vizcaíno, 2004); Maravillas del español (Chiquito, Naranjo, Gómez, Álvarez, \& Restrepo, 2012) con asesoría de la Universidad de Bergen en Noruega; Serie Enlace: Curso de español para extranjeros (Ariza, Beltrán, Forero, \& Páez, 2013); Usted tiene la palabra, niveles básico, intermedio y avanzado (JaramilloCardona, 2008, 2009, 2011).

Por último, los datos encontrados solo citan los nombres de libros de texto más representativos para el año y, en algunos casos, el origen de material; sin embargo, no se encontró información referente al programa o la institución educativa, por lo tanto, se llevó a cabo una revisión de los sitios web de los programas de ELE en el país, junto con encuestas dirigidas a coordinadores y docentes expertos y entrevistas focalizadas. 


\section{Recursos didácticos de ELE en Bogotá y Medellín}

Con el fin de de caracterizar los recursos didácticos utilizados en los programas de ELE en Bogotá y Medellín, el grupo investigador llevó a cabo encuestas y entrevistas a docentes, expertos o personas relacionadas con el campo ELE en estas ciudades. De esta manera, se solicitó la autorización a la coordinadora del programa de Maestría en español como Segunda Lengua y Lengua Extranjera, del Instituto Caro y Cuervo, la profesora Gloria Viviana Nieto, para enviar una encuesta sobre el uso de materiales en los programas de ELE pertenecientes al portal Spanish in Colombia en las ciudades objeto de estudio.

La encuesta fue realizada a coordinadores y profesores de programas de ELE, y contenía una serie de preguntas que buscaban conocer las percepciones de los docentes de ELE con respecto al uso del material didáctico en las clases. En este cuestionario se abordaron temas sobre las actividades, los materiales adicionales, las expectativas de los estudiantes, entre otros asuntos. A pesar de ser una herramienta que simplifica y optimiza la comunicación.En este ejercicio hubo pocas respuestas por parte de los docentes y de las instituciones que imparten cursos de ELE.

Adicionalmente, se sostuvo una reunión con una representante de la editorial Difusión para Bogotá, y otras ciudades del país, quien aceptó participar en el estudio y proporcionó datos sobre los libros de textos que se utilizan en los programas de ELE del país para el 2018.

En la entrevista se habló de los requerimientos y necesidades en torno a los materiales de ELE en el contexto local. De esta manera, el grupo investigador pudo conocer que, por una parte, otras universidades locales se encuentran en proceso de creación de su propio material ELE y, por otra, se identificaron los libros que soportan el programa curricular de los cursos de ELE ofrecidos en Bogotá y otras ciudades. 
Al confrontar todas las fuentes de información, primero, la revisión bibliográfica presentada anteriormente en el estado de la cuestión y en la información contenida en las páginas web, se obtuvo un grupo de materiales instruccionales para ELE utilizados entre el 2005 y el 2016, segundo la encuesta aplicada a IES parte del portal spanishincolombia y por último la entrevista realizada, se logró una aproximación al uso de materiales instruccionales para ELE utilizados mayormente utilizados en Bogotá y Medellín, consolidados en la tabla 1.

Tabla 1. Materiales instruccionales para ELE utilizados en Bogotá/Medellín 2017-2018.

\begin{tabular}{|c|c|c|}
\hline $\begin{array}{l}\text { Materiales } \\
\text { instruccionales } \\
\text { ELE }\end{array}$ & $\begin{array}{l}\text { Programa ELE - Institución Educativa } \\
\text { en Bogotá / Medellín }\end{array}$ & Comentarios \\
\hline Aula Internacional & $\begin{array}{c}\text {-Universidad Sergio Arboleda } \\
\text {-Pontificia Universidad Javeriana } \\
\text {-Universidad Central } \\
\text {-Instituto Caro y Cuervo (hasta 2018-1) } \\
\text {-Berlitz } \\
\text {-Universidad de los Andes (en estudio) }\end{array}$ & Variante dialéctica española \\
\hline Aula latina & $\begin{array}{l}\text { Se descontinuó en los programas de } \\
\text { Colombia. }\end{array}$ & $\begin{array}{l}\text { Alto contenido de la variante } \\
\text { dialéctica proveniente de México. }\end{array}$ \\
\hline Aula América & Instituto Caro y Cuervo - 2018 & $\begin{array}{l}\text { Recurso adaptado por el ICC } \\
\text { para contextos latinoamericanos, } \\
\text { publicación en el } 2018 .\end{array}$ \\
\hline Enlace & $\begin{array}{l}\text {-Universidad Externado de Colombia } \\
\text {-Universidad Santo Tomás }\end{array}$ & $\begin{array}{c}\text { Recurso creado por grupo } \\
\text { investigador de la universidad en } \\
\text { Bogotá }\end{array}$ \\
\hline Español al Vuelo & Universidad Pontificia Bolivariana & $\begin{array}{c}\text { Recurso creado por grupo } \\
\text { investigador de la universidad en } \\
\text { Medellín }\end{array}$ \\
\hline $\begin{array}{l}\text { Español al Vuelo } \\
\text { A2 }\end{array}$ & Universidad Pontificia Bolivariana & $\begin{array}{l}\text { Recurso creado por grupo } \\
\text { investigador de Medellín }\end{array}$ \\
\hline $\begin{array}{l}\text { Maravillas del } \\
\text { español }\end{array}$ & Universidad EAFIT & $\begin{array}{c}\text { Recurso creado por grupo } \\
\text { investigador de Medellín en asesoría } \\
\text { con la Universidad de Bergen, } \\
\text { Noruega. }\end{array}$ \\
\hline Gente & EAN & Variante dialéctica española \\
\hline Bitácora & $\begin{array}{l}\text { Universidad Distrital Francisco José de } \\
\text { Caldas - ILUD (en estudio) }\end{array}$ & Mayor enfoque léxico \\
\hline $\begin{array}{c}\text { Prisma / Prisma } \\
\text { Latino }\end{array}$ & $\begin{array}{l}\text { Se descontinuó en los programas de } \\
\text { Colombia }\end{array}$ & Se usa como material de apoyo \\
\hline $\begin{array}{l}\text { Usted tiene la } \\
\text { palabra }\end{array}$ & Universidad Pontificia Bolivariana & $\begin{array}{l}\text { Recurso creado por grupo } \\
\text { investigador de Medellín }\end{array}$ \\
\hline
\end{tabular}




\begin{tabular}{|c|c|c|}
\hline $\begin{array}{l}\text { Materiales } \\
\text { instruccionales } \\
\text { ELE }\end{array}$ & $\begin{array}{l}\text { Programa ELE - Institución Educativa } \\
\text { en Bogotá / Medellín }\end{array}$ & Comentarios \\
\hline Texto propio & $\begin{array}{c}\text {-Universidad de La Sabana (con apoyo } \\
\text { de otros textos) } \\
\text {-EAFIT } \\
\text {-Universidad Nacional }\end{array}$ & Variante dialéctica colombiana \\
\hline $\begin{array}{c}\text { Otro tipo de } \\
\text { recurso de apoyo }\end{array}$ & Politécnico Grancolombiano & $\begin{array}{c}\text { No llevan libro guía, solo uso de } \\
\text { plataforma virtual }\end{array}$ \\
\hline
\end{tabular}

Fuente: elaboración propia, resultado de revisión bibliográfica, entrevistas y encuestas realizadas por el grupo investigador.

De la entrevista también se pudo conocer que la Editorial Difusión se encuentra impulsando dos nuevas líneas de materiales didácticos ELE: material instruccional para clases personalizadas, en atención a las necesidades de los cursos locales, y un campus digital cargado de diverso tipo de recursos digitales y fotocopiables para ser utilizados en el aula ELE. Para acceder al "CampusDifusion. com", se debe adquirir una licencia de acceso por un determinado periodo de tiempo.

\section{Análisis interpretativo de los recursos de ELE a través de la triangulación hermenéutica}

En la fase de la revisión de textos a través de la matriz documental, los investigadores siguieron el formato diseñado para este instrumento (anexo 1) y, de esta manera, consignaron la información relacionada con las características de los textos. Los textos analizados fueron escogidos por ser los que comúnmente se utilizan en las clases de ELE. Los aspectos que se tuvieron en cuenta para la recolección y el análisis fueron la estructura del texto, las actividades, el manejo de las imágenes, los contenidos, entre otros. Por último, la matriz documental de los recursos didácticos siguió un proceso similar al de recolección y análisis de los textos de ELE; sin embargo, algunos ítems de análisis tuvieron que ser modificados 
dada la caracterización de los recursos didácticos. Luego de haber consignado la información en los formatos correspondientes, los investigadores consolidaron los hallazgos. Esta información se presentará en la siguiente sección.

\section{Ruta metodológica}

La ruta metodológica para el desarrollo de esta investigación inició con la selección y revisión de los recursos didácticos empleados en las clases de ELE en las universidades de Bogotá y Medellín, que se analizaron a través del diseño de una matriz categorial que permitió identificar sus principales características y relacionarlas con el objeto de estudio. Este estudio también incluyó instrumentos de recolección de la información como entrevistas semiestructuradas y diarios de campo, a partir de la triangulación hermenéutica, que se define como "la acción de reunión y cruce dialéctico de toda la información pertinente al objeto de estudio surgida en una investigación, por medio de los instrumentos correspondientes" (Cisterna-Cabrera, 2005, p. 68).

\section{Análisis documental de libros}

A través de la tabla de análisis documental de libros, el grupo investigador principalmente buscó tener una percepción más exacta de cómo el uso de los textos para ELE y su estructura aportan a los procesos de enseñanza y aprendizaje del español. Al igual que el análisis de los otros estamentos (los estudiantes, los docentes y los expertos), los resultados mostrados por la matriz categorial les dieron a los investigadores una idea clara de cómo es la estructura de los textos y hasta qué punto esta información aporta a la respuesta de las preguntas de investigación y a la consecución de los objetivos del proyecto. Cabe anotar que este análisis se hizo sobre los textos más comunes utilizados en las clases de ELE, lo que da una total validez a este instrumento de recolección de información. 
A continuación, se hace una descripción de los aspectos más importantes identificados en los textos analizados.

\section{Caracterización de los recursos didácticos empleados en los programas de ELE}

En primera instancia, los textos son claros en cuanto a los objetivos de aprendizaje que tendrán los usuarios a lo largo del contenido, al igual que los referentes internacionales sobre los cuales se basan los textos. Esta información es de gran importancia, ya que los usuarios conocen, desde el inicio de su proceso, los objetivos que deben alcanzar y el nivel de salida que propone el Marco Común Europeo de Referencia para las lenguas (MCERL). Con respecto al desarrollo de competencias, los textos analizados enfocan sus prácticas en alto grado hacia el desarrollo de las competencias pragmáticas y la sociolingüística, dado que estas otras competencias fortalecen en el estudiante la capacidad para utilizar el idioma en contextos reales y significativos.

Con relación a los estilos de aprendizaje, algunas de las actividades propuestas en los textos, tienen como objetivo enfrentar al usuario a diversas situaciones comunicativas en las que tenga herramientas suficientes para establecer procesos exitosos de comunicación; además, los textos proponen varias estrategias de aprendizaje para que, según sus perfiles, los usuarios puedan utilizarlas y, así, optimizar su proceso de aprendizaje. Algunas de las estrategias propuestas en los textos son las siguientes:

- Utilización de imágenes para memorizar categorías de vocabulario.

- Ejercicios escritos en los que los estudiantes deben identificar las categorías estructurales y los componentes de las oraciones. 
- Ejercicios de emparejamiento.

- Ejercicios de interacción y trabajo independiente.

Asimismo, los textos proveen diversos estilos de aprendizaje para ofrecerles a los estudiantes alternativas al momento de aprender y de potencializar su proceso. Los más predominantes son el estilo visual y el auditivo: en cuanto al estilo visual, se puede afirmar que los textos son muy ricos en cuanto a las imágenes, posiblemente por hacer del texto un material agradable y atractivo para los estudiantes; en cuanto al estilo auditivo, los estudiantes van a familiarizar su oído más rápidamente con la lengua de salida (español). Finalmente, los estilos social e individual se presentan como alternativas de interacción y trabajo independiente.

Otro de los aspectos representativos con respecto al proceso de aprendizaje es la oportunidad que tienen los usuarios para reflexionar y desarrollar el pensamiento crítico. En todos los textos se incluyen ejercicios que les permiten a los estudiantes reflexionar sobre sus gustos, afinidades y lo que no les gusta. Además, pueden establecer diferencias y contrastes entre aspectos sociales o culturales de los países en donde se habla la lengua que están aprendiendo con su lengua nativa. Se considera que estas actividades ayudan en gran medida a que los usuarios no vean la lengua española como un ente meramente estructural, sino también como un verdadero puente de comunicación en el que se consideren aspectos personales, culturales y contextuales de varias partes del mundo.

Con respecto al material que traen los textos para el docente, es necesario hacer dos especificaciones que, directa o indirectamente, influyen en el proceso de aprendizaje de los usuarios. Primero, ningún texto trae material adicional para el docente; esto hace que, aunque los textos sean completos y facilitadores del proceso de aprendizaje, los instructores deban diseñar y tener material adicional para complementar sus prácticas y acercar a los estudiantes a un proceso significativo de aprendizaje. Segundo, los textos cuentan con recursos de tipo pedagógico y auténtico. Estas dos 
características hacen que el estudiante tenga la oportunidad de enfrentarse a diferente tipo de material auditivo y escrito. Es importante destacar que, según las propias percepciones de los estudiantes, el hecho de tener material propio de la lengua española, sin adaptaciones pedagógicas, crea en el usuario un reto mayor para comprender el material al que están expuestos.

El último aspecto que el grupo investigador considera esencial en el análisis de los textos seleccionados es el contenido cultural que traen los textos para las prácticas docentes. En relación con la inclusión del componente cultural, todos los textos lo incorporan, lo que hace que los usuarios tengan un contacto casi que directo y real con diferentes aspectos culturales de países en donde la lengua materna es el español. Si bien es cierto que se nota un gran esfuerzo por considerar temas de América Latina, los textos tienen una marcada tendencia española en cuanto a aspectos como la música, la comida, los sitios turísticos, las costumbres, entre otros. Esto significa que la mayor parte del tiempo y del curso, los estudiantes van a estar expuestos a información cultural de España. Además, es interesante tener en cuenta para el desarrollo de la competencia cultural en las clases de ELE, a partir de los textos, hasta qué punto estos establecen diferencias culturales que los estudiantes puedan identificar. Como respuesta a esta inquietud, se podría afirmar que, efectivamente, los textos sí contrastan la cultura española y la cultura latinoamericana de manera general $y$, además, implementan varias estrategias para hacerlo: ejercicios de interacción entre estudiantes; cuadros de contraste entre dos culturas; formulación de preguntas y respuestas, entre otras.

Finalmente, con respecto a la inclusión del componente cultural colombiano, se pudo establecer que solamente un texto de los que se analizaron abordó este aspecto, lo que evidencia una clara ausencia de este tipo de material para los docentes que se encuentren en el contexto colombiano. Considerando la información suministrada, se puede concluir que los textos que se usan en las clases de ELE poseen una serie de características pedagógicas, lingüísticas y pragmáticas que, en cierta manera, garantizan que el usuario tenga un acercamiento real a la lengua española, y traba- 
jan todas las destrezas comunicativas, no solo estructurales, sino contextuales, que eventualmente benefician el proceso de aprendizaje de los estudiantes. Sin embargo, carecen de un acercamiento significativo y efectivo a la cultura colombiana que les dé a los usuarios la oportunidad de conocer más sobre el entorno en el que están aprendiendo el idioma.

\section{Hallazgos sobre los recursos didácticos ELE}

De todas las fuentes de información analizadas, se puede afirmar que, aunque ha habido un incremento en el diseño de materiales con contenido cultural o dialectal colombiano en el país, aun son pocos los programas que los incluyen como herramienta principal de aprendizaje.

Por otra parte, en relación con la forma y contenido de los recursos didácticos de ELE, al realizar la revisión documental y al revisar lo mencionado por los estamentos docentes, expertos y estudiantes, podemos decir que son materiales cuyo enfoque parte del desarrollo de competencias para el aprendizaje de las lenguas extranjeras y, para lograrlo, se establecen actividades encaminadas a trabajar de manera integral las competencias lingüística, pragmática y sociolingüística. Como consecuencia de esto, los recursos didácticos analizados cuentan con gran variedad de actividades en las que los estudiantes pueden utilizar varios estilos de aprendizaje, convirtiéndose así en material indispensable para la práctica docente y para el alcance de los objetivos de aprendizaje.

La variedad de actividades propuestas cuentan, además, con un propósito que va más allá del netamente curricular, que es precisamente promover, estimular y propiciar el desarrollo del pensamiento crítico en los estudiantes. Esto se logra a través del uso contextualizado de la lengua objeto: el español. Por otra parte, los recursos didácticos incluyen diferentes fuentes de información; es decir, el material presentado puede comprender actividades dise- 
ñadas por el docente o material pedagógico que se adapta a las necesidades de los estudiantes y a los objetivos establecidos para las sesiones. Adicionalmente, los recursos también se han visto impactados por el avance de las tecnologías y no solo se realizan guías de trabajo, sino que se incluyen actividades de Internet, que van desde sitios de práctica hasta aquellos como juegos, imágenes e información cultural.

\section{Otros recursos utilizados en}

las aulas de ELE en Colombia

Los recursos didácticos seleccionados hacen parte del material complementario con el que cuentan los profesores para el desarrollo de sus sesiones de ELE. Se puede decir, como un primer punto de esta descripción, que tanto los objetivos de aprendizaje del material como los referentes internacionales son claros y explícitos. De esta manera, los usuarios pueden saber qué objetivos deben cumplir y la relación curso-nivel de salida MCER. Igualmente, en cuanto al desarrollo de competencias para el aprendizaje de las lenguas extranjeras, los recursos hacen uso, no solamente de la competencia lingüística, sino también de las competencias pragmáticas y sociolingüísticas, con el propósito de involucrar al estudiante en diversos contextos de aprendizaje y guiarlo hacia el desarrollo de procesos efectivos de comunicación. En general, los recursos didácticos analizados cuentan con gran variedad de actividades en las que los estudiantes pueden utilizar diversos estilos de aprendizaje (individual, visual, auditivo e intrapersonal).

Las actividades propuestas en los recursos promueven el pensamiento crítico en los usuarios, de tal manera que puedan utilizar la lengua como medio de comunicación y como recurso para expresar sus percepciones sobre los contextos en los que se habla el español. Los materiales analizados son ricos en cuanto a la tipología que manejan. Hay actividades diseñadas con material pedagógico y con material auténtico que, a su vez, resulta más complejo para los estudiantes. Los recursos encontrados van desde guías de 
trabajo elaboradas para el desarrollo de determinado tema, hasta sitios de Internet a través de los cuales los estudiantes pueden encontrar diferentes actividades y ejercicios.

Un factor similar al análisis de los textos es la inclusión del componente cultural pues, aunque sí se incluye en estos recursos, tiene una fuerte influencia española que podría limitar el espectro de aprendizaje de los estudiantes; es decir, se estarían limitando a solamente una fuente de origen. Los recursos didácticos establecen un contraste entre los componentes culturales españoles y latinoamericanos, aunque se evidenció que en algunos casos se hizo de manera superficial. Con respecto a la inclusión del componente cultural colombiano, el análisis muestra que no se incluye, salvo información aislada que presentan algunos recursos como nombres de ciudades y algún sitio turístico representativo. Esta es una característica en común de los recursos analizados. Además, esto permite concluir que, si los docentes desean abordar la cultura colombiana, lo deben hacer con material propio diseñado por ellos.

Finalmente, un aspecto para resaltar en esta sección es la inclusión de material digital en el proceso de aprendizaje de los estudiantes. La plataforma digital analizada, cuya ventaja más importante es el aprovechamiento y la sistematización del trabajo independiente, ofrece una amplia gama de ejercicios que permiten que el usuario practique constantemente la lengua. Hay gran variedad de ejercicios de lectura, estructura, ejercicios de comprensión auditiva y de vocabulario. Se considera como una herramienta muy apropiada para el desarrollo del trabajo independiente; no obstante, este recurso no aborda de manera significativa el componente cultural colombiano.

Tendencias y aproximaciones culturales en los recursos analizados hallazgos

Una de las características más importantes de los recursos es la inclusión del componente cultural. El inconveniente radica en que, 
en su mayoría, se trata de material relacionado con la cultura española y el aspecto latinoamericano se aborda de manera superficial (a excepción de un caso, el contexto colombiano no aparece visible). Por lo tanto, los docentes deben acudir a material diseñado por ellos o a fuentes de información originales que los ayuden a contrastar la cultura de los estudiantes con el contexto colombiano. Esta falta de recursos didácticos, que incluyan el componente cultural colombiano, es una de las debilidades señaladas por el estamento docente, expertos y estudiantes, ya que afirman que, si bien es cierto que es una necesidad latente en las clases de ELE, la falta de material disponible lo convierte en todo un reto a la hora de incluirlo en las sesiones.

Con respecto a qué materiales contribuyen a la inclusión del componente cultural colombiano, se encontró que la mayoría de los libros de texto y recursos didácticos no cuentan con guías de ELE con inclusión de la cultura colombiana y que los docentes entrevistados deben adaptar y diseñar este tipo de recursos, que pueden incluir lecturas, videos y canciones de material auténtico colombiano. Cabe anotar que este material, al momento del estudio, no estaba sistematizado, por lo que no era accesible a la comunidad académica de ELE.

En cuanto al aporte que hicieron los docentes y expertos a la problemática de la ausencia de material con componente cultural colombiano, sugieren que, si se va a crear un recurso didáctico con estas características, se tenga en cuenta la importancia de términos como cultura, interculturalidad y transculturalidad, ya que este será un punto de inicio para el diseño y la implementación de las actividades. Es decir, se debe recordar que la cultura está dada no solo por aspectos extrínsecos como las comidas, la música, los sitios turísticos, etc., sino que debe ir más allá; se deben tratar temas no tan evidentes como la historia, la religión y todos aquellos aspectos que caracterizan cada cultura como algo único y diferenciable. Sugieren, además, no olvidar desde dónde y para quiénes se crea el recurso didáctico; es decir, tener presente los documentos institucionales como el PEl de las instituciones, el plan de desarrollo y la política curricular, ya que estos documentos son precisamente 
los que le permitirán a la propuesta tener un carácter diferenciador de las propuestas que actualmente se encuentran en el mercado, dando como resultado un producto único y enriquecedor en el campo de la enseñanza y aprendizaje del ELE.

\section{Conclusiones}

Es preciso resaltar que los recursos didácticos son una herramienta útil y efectiva para que los usuarios desarrollen las habilidades de la lengua, pues a través de ellos tienen la oportunidad de ahondar en sus debilidades y hacerlo a su propio ritmo, con las estrategias que les resulten más efectivas. Sin embargo, el grupo considera que la no inclusión del componente cultural colombiano podría incidir en que los estudiantes desconozcan varios aspectos importantes y representativos de nuestro contexto.

En relación con los recursos didácticos analizados en la revisión documental, se puede concluir que los libros de texto guía utilizados en los programas de ele en Bogotá y Medellín son, en su mayoría, diseñados por grupos editoriales internacionales. Todos los libros cumplen con estándares internacionales en cuanto a la presentación de objetivos curriculares, desarrollo de competencias lingüísticas, pragmáticas y sociolingüísticas para el aprendizaje de las lenguas extranjeras, ayudas pedagógicas digitales, entre otros. Con respecto al componente cultural, la mayoría de los libros incluye un alto contenido de actividades, recursos didácticos y vocablos de la cultura española. Pero a pesar de los esfuerzos en el país por la creación de materiales con contenidos de la cultura colombiana, solamente se encontraron tres libros de texto con este contenido al momento del estudio.

Desde la matriz de los recursos didácticos analizados, se encontró que estos materiales se orientan principalmente a la práctica lúdica de vocabulario y de producción oral, con muy bajo contenido cultural y sin ningún contenido cultural colombiano. Por otra parte, en los recursos diseñados o adaptados por los docentes partici- 
pantes, se encontraron materiales auténticos como videos, canciones y lecturas. Adicionalmente, los recursos analizados tienen una tendencia marcada a tratar aspectos visibles o superficiales de la cultura, y solamente algunos materiales de lectura incluyen temas más profundos, como los religiosos o históricos.

En la revisión del estado de la cuestión, se encontraron otros libros de enseñanza de ELE con inclusión de componente cultural colombiano; sin embargo, estos libros no son usados en los programas de ELE de las universidades locales, sino como material de apoyo o para programas de turismo.

Los investigadores de este proyecto coinciden en que el estado de la cuestión que aquí se presenta muestra claramente el hecho de que, aunque existen numerosos estudios y trabajos académicos con respecto al campo de ELE en Bogotá y en Colombia, es muy poco lo que se encuentra en relación con la inclusión del componente cultural colombiano. De allí, que este estudio tenga como objetivo principal no solo la caracterización de las prácticas docentes de ELE en las instituciones en las que se llevó a cabo y de los recursos utilizados en dichas prácticas, sino también la propuesta de un recurso didáctico que pueda suplir ese vacío identificado a lo largo del análisis teórico hecho, como parte fundamental del proyecto.

\section{Referencias bibliográficas}

Agencia Presidencial de Cooperación Internacional. (2013-2018). ELE Colombia: Programa Focalae. Bogotá: Gobierno de Colombia. Recuperado de https://www.apccolombia.gov.co/pagina/ele-focalae

Agray, N., \& Rico, C. (2015). Bogotá Vívela en español. Recuperado de http://bogotaturismo.gov.co/sites/default/files/bogota_vivela_baja2. pdf

Ariza, E., Beltrán, C., Forero, C., \& Páez, C., (2013). Enlace: Curso de español para extranjeros. Bogotá: Universidad Externado de Colombia. 
Beltrán, L. (2015). Laura Beltrán Arias. Influencia cultural en la motivación para el aprendizaje del inglés como lengua extranjera. Revista Interamericana de Investigación, Educación y Pedagogía, RIIEP, 8(2). DOI: https://doi.org/10.15332/25005421

Cabeza, S., Lobo, Vizcaíno. (2004). Cartagena Hispana y Multicultural, texto de español para estudiantes extranjeros: niveles básico, intermedio y avanzado. Cartagena: Universidad Tecnológica de Bolívar.

Calderón-Rivera, A. (2011). Español para extranjeros. Bogotá: Universidad Distrital Francisco José de Caldas.

Cardona, A., Gutiérrez, C., Delgadillo, C., \& Rodríguez, E. (2014). De paseo por Colombia: Material de apoyo para estudiantes de español como lengua extranjera. Bogotá: Ediciones Lengua y Cultura.

Cardona-Serrano, A. (2009). Gramática General del español como lengua extranjera. Bogotá: Ruiz Hermanos Impresores.

Cisterna-Cabrera, F. (2005). Categorización y triangulación como procesos de validación del conocimiento en investigación cualitativa. Theoria, 14(1), 61-71.

Continuum. (2014). Entrevistas: Todoele desde dentro. Wordpress. Recuperado de https://ccontinuumeledu.wordpress.com/entrevistas/ todoele-net-desde-dentro/

Chiquito, A., Naranjo, J., Gómez, R., Álvarez, D., \& Restrepo, M. (2014). Maravillas del español. Medellín: Universidad EAFIT.

Díaz, A. (2016). Estado del arte del español como lengua extranjera (ELE) en Bogotá: cursos de ELE que nacen y se hacen. Voces y Silencios. Revista Latinoamericana de Educación, 7(1), 173-193. doi: http://doi.org/c89v

García, D., García, J., \& Buitrago, Y. (2011). Estado del arte de ELE en Colombia, una mirada holística: Metodologías y enfoques, material Didáctico, variedades vingüísticas y dificultades en el área. MarcoELE. Revista de Didáctica Español Lengua Extranjera, 13, 1-10. Recuperado de http://www.redalyc.org/articulo.oa?id=92152421003 
Gonzáles. (2010). Enseñanza del español como lengua extranjera: Bibliografía comentada (Tesis de Maestría). Bogotá: Instituto Caro y Cuervo.

Instituto Cervantes. (2018). El español: una lengua viva. Informe. Madrid: Instituto Cervantes. Recuperado de https://cvc.cervantes.es/ lengua/espanol_lengua_viva/pdf/espanol_lengua_viva_2018.pdf

Jaramillo, G. (2008). Usted Tiene La Palabra: nivel básico. Medellín: Universidad Pontificia Bolivariana.

Parr-Giraldo, M., \& Rojas-Bernal, L. (2007). Comuniquémonos en español. Bogotá: Editorial Magisterio.

Presidencia de la República. (2010). Plan General de Desarrollo 20102014. Consultado el 7 de Marzo de 2016. Recuperado de https:// colaboracion.dnp.gov.co/CDT/PND/Resumen $\% 20$ Ejecutivo $\% 20$ Ultima\%20Version.pdf

Reyes-Angona, Sergio (2016). El aprendizaje en una comunidad en internet de profesores de español como segunda lengua: estudio etnográfico virtual de la comunidad Todoele (Tesis Doctoral). Universidad Complutense, Madrid, España. Recuperado de https://eprints.ucm. es/40462/1/T38126.pdf

Rojas, N. (2017). Vamos a turistear aprendiendo español: Propuesta didáctica para el aprendizaje de léxico y expresiones durante el recorrido turístico en la Catedral de Sal de Zipaquirá (Tesis de Maestría). Pontificia Universidad Javeriana, Bogotá, Colombia. Recuperado de https:// repository.javeriana.edu.co/handle/10554/34378

Tahull J.(2016). Modernidad, educación y género. El proyecto inacabado Revista Interamericana de Investigación, Educación y Pedagogía, RIIEP, 9(2). DOI: https://doi.org/10.15332/25005421

Velandia, D., Ussa, M., \& Waked M. (2008-2015). Español para extranjeros: niveles inicial, intermedio y avanzado. Tunja: Universidad Pedagógica y Tecnológica de Colombia.

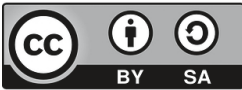




\section{Anexos}

Anexo 1. Matriz documental de textos y recursos didácticos.

\begin{tabular}{|c|c|c|}
\hline Parámetro a analizar & Libro 1 & Libro: 2 \\
\hline \multicolumn{3}{|l|}{ Currículo } \\
\hline \multicolumn{3}{|l|}{$\begin{array}{l}\text { Objetivos de aprendizaje: Se establecen y } \\
\text { cómo se enuncian }\end{array}$} \\
\hline \multicolumn{3}{|l|}{$\begin{array}{l}\text { Contenidos: Referentes desde dónde se } \\
\text { establecen y categorias presentadas }\end{array}$} \\
\hline \multicolumn{3}{|l|}{ Correspondencia MCER: Si o No } \\
\hline \multicolumn{3}{|l|}{$\begin{array}{l}\text { Competencias de las Lenguas Extranjeras: } \\
\text { Comunicativa: Pragmática, Lingüística y } \\
\text { sociolingüística }\end{array}$} \\
\hline \multicolumn{3}{|l|}{ Proceso de aprendizaje } \\
\hline \multicolumn{3}{|l|}{$\begin{array}{c}\text { Estrategias de aprendizaje: 1) Directas: } \\
\text { cognitivas, compensación, memoria. 2) } \\
\text { Indirectas: Meta cognitivas, sociales y } \\
\text { afectivas }\end{array}$} \\
\hline \multicolumn{3}{|l|}{$\begin{array}{l}\text { Estilos de aprendizaje: visual, auditivo, } \\
\text { kinestésico, táctil, social, individual }\end{array}$} \\
\hline \multicolumn{3}{|l|}{$\begin{array}{l}\text { Pensamiento crítico: conocimiento, } \\
\text { comprensión, aplicación y análisis }\end{array}$} \\
\hline \multicolumn{3}{|l|}{$\begin{array}{c}\text { ¿La serie trae material didáctico para el } \\
\text { docente? }\end{array}$} \\
\hline \multicolumn{3}{|l|}{ Tipología de los recursos } \\
\hline \multicolumn{3}{|l|}{ Inclusión componente cultural } \\
\hline \multicolumn{3}{|l|}{ Desarrollo de la competencia intercultural } \\
\hline \multicolumn{3}{|l|}{ Diferencias culturales } \\
\hline \multicolumn{3}{|l|}{ Componente cultural colombiano } \\
\hline \multicolumn{3}{|l|}{$\begin{array}{l}\text { Recursos para el desarrollo del componente } \\
\text { cultural en las sesiones ELE }\end{array}$} \\
\hline Valor agregado del texto & & \\
\hline
\end{tabular}

Fuente: elaboración propia 